Recepción: 20 / 04 / 2017

Aceptación: 20 / 05 / 2017

Publicación: 15 / 07 / 2017

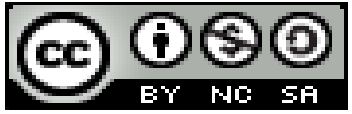

Ciencias de la Salud

Artículo Científico

\title{
El Cáncer de ovario y la efectividad del HE4 como Biomarcador Tumoral
}

\author{
Ovarian Cancer and the effectiveness of HE4 as a Tumor Biomarker
}

Cancro do ovário e HE4 eficácia como um biomarcador tumoral

Esthela N. Tinoco-Moreno ${ }^{\mathrm{I}}$ esthela.tinocom@ug.edu.ec

Alex F. Vivas-Lara ${ }^{\text {II }}$ alex.vivas1@ug.edu.ec

María G. Acuña-Chong III maria.acuñach@ug.edu.ec

Mónica G. Núñez-Nájera IV mona.sanmiguel@gmail.com

Correspondencia: esthela.tinocom@ug.edu.ec 


\section{Resumen}

El interés en la detección temprana del cáncer de ovario para lograr la reducción de la mortalidad ha crecido, con el descubrimiento de biomarcadores tumorales séricos asociados a tumores malignos. El estudio realizado evalúa 53 pacientes entre abril 2015 y marzo 2016 con masas pélvicas, incluyendo $32(60,4 \%)$ mujeres post menopáusicas y $21(39,6 \%)$ mujeres pre menopáusicas. Valores de sensibilidad, especificidad, valor predictivo positivo y negativo (VPP, VPN), razón de probabilidad positiva y negativa (LR+, LR-), y pruebas estadísticas para determinar la relación entre los estados menopáusicos, y los grupos de estudio (benigno, maligno y control) de CA125, HE4 y ROMA fueron calculados con un intervalo de confianza del 95\%. Todo resultado con nivel de significancia $\mathrm{p} \leq .05$ fue aceptado como estadísticamente significante. HE4 presentó un valor medio diferenciable que permite distinguir masas pélvicas malignas de benignas (Log2 HE4:7.19 (maligno) vs. 5.71 (benigno); control 5.79), igualmente, HE4 + ROMA presentan mayor sensibilidad y especificidad que las combinaciones CA125 + HE4 y CA125 + I. ROMA (S: 86\% vs. 64\%; E:97\% vs. 93\%, respectivamente), de igual forma HE4 + ROMA presentan mayores niveles de VPP, VPN, LR+ y la menor LR- (95\%, 91\%, 27.43 y 0.15, respectivamente). En conclusión, los resultados sugieren que HE4 y ROMA en combinación o solos, servirían como biomarcadores eficientes para la diferenciación de masas pélvicas en estadíos tempranos y si se adiciona el estatus menopaúsico, afianza los resultados obtenidos, resultando un algoritmo diagnóstico idóneo, para la diferenciación del cáncer de células epiteliales en estadios tempranos.

Palabras claves: Biomarcador; cáncer de ovario; especificidad; sensibilidad. 
Esthela N. Tinoco-Moreno; Alex F. Vivas-Lara; María G. Acuña-Chong; Mónica G. Núñez-Nájera

\section{Summary}

Interest in the early detection of ovarian cancer to achieve mortality reduction has grown with the discovery of tumoral biomarkers associated with malignant tumors. The study evaluated 53 patients between April 2015 and March 2016 with pelvic masses, including 32 (60.4\%) postmenopausal women and $21(39.6 \%)$ premenopausal women. Sensitivity, specificity, positive and negative predictive value (PPV, NPV), positive and negative likelihood ratio (LR +, LR-), and statistical tests to determine the relationship between menopausal status and study groups (benign, malignant and control) of CA125, HE4 and ROME were calculated with a 95\% confidence interval. Any result with level of significance $\mathrm{p} \leq .05$ was accepted as statistically significant. HE4 presented a mean differentiable value that allows to distinguish malignant pelvic masses from benign (Log2 HE4: 7.19 (malignant) vs. 5.71 (benign), control 5.79), also, HE4 + ROMA present greater sensitivity and specificity than the combinations CA125 + HE4 and (95\% vs. 64\%, E: 97\% vs. 93\%, respectively). Similarly, HE4 + ROMA had higher levels of PPV, NPV, LR + and the lowest LR- (95\%, 91\%, 27.43 and 0.15 , respectively). In conclusion, the results suggest that HE4 and ROME in combination or alone, would serve as efficient biomarkers for the differentiation of pelvic masses in early stages and if the menopausal status is added, it consolidates the results obtained, resulting in a suitable diagnostic algorithm for differentiation Of epithelial cell cancer in early stages

Key words: Biomarker; ovarian cancer; specificity; sensitivity. 
Esthela N. Tinoco-Moreno; Alex F. Vivas-Lara; María G. Acuña-Chong; Mónica G. Núñez-Nájera

\section{Resumo}

O interesse na detecção precoce do cancro do ovário para alcançar a redução da mortalidade tem crescido com a descoberta de marcadores tumorais no soro associados a tumores malignos. O estudo avaliou 53 pacientes, entre Abril de 2015 e em março 2016 com massas pélvicas, incluindo 32 $(60,4 \%)$ e 21 mulheres na pós-menopausa $(39,6 \%)$ mulheres na pré-menopausa. Sensibilidade, especificidade, valor preditivo positivo e negativo (PPV, NPV), porque probabilidade negativo positivay $(\mathrm{LR}+, \mathrm{V}-)$ e testes estatísticos para determinar a relação entre os estados da menopausa e grupos de estudo (benignas, malignas e controlo) CA125, HE4 e cigana foram calculados com um intervalo de confiança de 95\%. Qualquer resultado com nível de significância de $\mathrm{p} \leq 0,05$ foi aceito como estatisticamente significativo. HE4 tinha um valor médio diferenciável que distingue massas pélvicas benignas malignas (Log2 HE4: 7,19 (malignas) versus 5,71 (benigno); 5,79 Controlo) do mesmo modo HE4 + Roma têm maiores combinações de sensibilidade e especificidade de CA125 + HE4 e (: $86 \%$ versus 64\%; e: S 97\% vs. 93\% respectivamente) I. ROMA CA125 +, semelhante HE4 + Roma têm níveis mais elevados de PPV, NPV, LR + e LR- inferior (95\% , 91\%, 27,43 e 0,15, respectivamente). Em conclusão, os resultados sugerem que HE4 e cigana em combinação ou sozinhos, servir como biomarcadores eficazes para a diferenciação de massas pélvicas nas fases iniciais e se o estado de menopausa é adicionado, consolida os resultados obtidos, o que resulta em um diagnóstico apropriado algoritmo de diferenciação cancro de células epiteliais no início

Palavras-chave: Biomarcador; cancro do ovário; a especificidade; a sensibilidade. 


\section{Introduccion}

Los tumores de ovario son un grupo de neoplasias diversas por su epidemiología, histogénesis e historia natural. Entre 2009 y 2013, 215.500 a 238.000 nuevos casos de cáncer de ovario fueron diagnosticados mundialmente y 146.000 a 152.000 mujeres murieron por esta causa. El cáncer de ovario epitelial (EOC) es una de las principales causas de mortalidad femenina asociado a su ginecología. La tasa de mortalidad reportada según Solca (2012) en la ciudad de Guayaquil es de 34 mujeres, con una incidencia de tasa estandarizada de 6.3/100,000 habitantes. La etiología del EOC se considera que probablemente sea de carácter multifactorial, sin embargo, se considera que los factores más asociados a esta enfermedad serían: edad, obesidad, antecedentes relacionados con la reproducción, estatus menopáusico, antecedentes familiares de cáncer de ovario, mama y cáncer colorectal, entre otros.

El cáncer de ovario aunque tiene baja prevalencia es considerado entre las malignidades ginecológicas más letales por su alta mortalidad (Wilailaket al., 2015), siendo el segundo tumor maligno ginecológico y el octavo cáncer más frecuente en la mujer: de cada cien tumores malignos en mujeres, tres son cáncer de ovario. Entre 2009 y 2013, 215.500 a 238.000 nuevos casos de cáncer de ovario fueron diagnosticados mundialmente y de 146.000 a 152.000 mujeres murieron por esta causa (Ferlayet al., 2013; Jernalet al., 2009).

Debido al incremento del número de mujeres con cáncer de ovario que son referidas a especialistas, rangos de predicción de riesgos han sido desarrollados para identificar mujeres con riesgo alto de cáncer de ovario mediante, la incorporación de datos clínicos (ej. estatus menopáusico), imagenología (ej. ultrasonido) y/o aplicación de biomarcadores tumorales (ej. Antígeno cancerígeno 125 (CA125), proteína epididímica humana 4 (HE4) en modelos regresivos. 
Ejemplos de modelos de predicción de riesgo incluyen: (1) Índice de Riesgo de Malignidad (RMI, por sus siglas en ingles) (Jacobset al., 1990); (2) Algoritmo de Riesgo de Malignidad Ovárica (ROMA, por sus siglas en ingles) el cual utiliza estatus menopáusico más valores de CA125 y HE4 (Moore et al., 2009); OVA1 el cual utiliza CA125, beta 2 microglobulina, apolipoproteína A1, Ferritina y Transferrina (Abraham, 2010) y (4) LR2 un modelo de predicción ultrasónico desarrollado por International Ovarían Tumor Analysis (IOTA) (Nuneset al., 2012).

Varios trabajos científicos han identificado a HE4 como un marcador para cáncer ovárico con mejorada especificidad en relación a CA125 (Escudero et al., 2011). Este biomarcador tumoral HE4 es una proteína presente en altas concentraciones en el epidídimo en hombres y también se encuentra en pacientes con cáncer de ovario (Gaoet al., 2011).

Con este estudio de caso se pretende determinar la eficacia del uso del biomarcador HE4 para la detección precoz de cáncer epitelial de ovario en estadíos tempranos, lo cual combinado con chequeos ginecológicos e incluyendo el biomarcador CA125 y/o índice ROMA, sirvan como herramienta diagnóstica eficaz que ayude a disminuir y controlar eficientemente el avance de esta enfermedad, que afecta fuertemente a la población.

\section{Resultados}

- $\quad$ El estudio verifico que con masas pélvicas, el $60,4 \%$ mujeres eran pos menopáusica y el $39,6 \%$ de mujeres pre menopáusicas. Del total, el 22,6\% presentaban tumoraciones benignas, el 39,6\% tenían EOC y el 37,8\% correspondían a pacientes control. La edad media (segundo percentil) de las pacientes con tumores benignos fue de $38,0 \pm 10,45$ años, la media de edad en el primer 
percentil fue de 30,50 \pm 9,11 años, la media de edad en el tercer percentil fue de 42,00 \pm 4,41 años, obteniendo un rango inter cuartílico de (RIC 11,50). Los valores respectivos para las pacientes con tumores malignos y el grupo control respectivamente fueron 55,00 \pm 19,66 años, 31,00 $\pm 13,74$ años, 63,00 \pm 7,40 años, obteniendo un rango inter cuartílico de (RIC 32,00); Grupo control 46,00 \pm 18,18 años, 36,00 \pm 9,06 años, 63,00 \pm 9,43 años, obteniendo un (RIC 27,00).

- Las valoraciones de los tumores difieren en mujeres pre menopáusicas y pos menopáusicas separadamente. Los niveles de CA125 difieren significativamente entre tumoraciones benignas versus malignas ( $p: 0.012957)$ y en malignas versus el grupo control ( $p$ : 0.023288). No presentan diferencia significativa entre las tumoraciones benignas y el grupo control. HE4 difiere significativamente entre las tumoraciones benignas versus malignas ( $p$ : 0.018363). No existe diferencia significativa entre benignas versus control y malignas versus control; ROMA no presentó diferencia significativa en ninguna de las comparaciones. Los niveles de los biomarcadores tumorales difieren significativamente entre las mujeres con masas benignas y las diagnosticadas con cáncer de ovario, frente al grupo control.

- Los niveles de los biomarcadores tumorales difieren significativamente entre las mujeres con masas benignas y las diagnosticadas con cáncer de ovario, frente al grupo control. Los valores medios de los marcadores HE4 y CA125 así como el índice ROMA de las mujeres diagnosticadas con cáncer de ovario difieren fuertemente frente al control (CA125: 5,72 \pm 2,77; HE4: 7,19 \pm 1,57; I.ROMA: 2,42), existiendo rangos diferenciales de biomarcador en las mujeres que presentan tumoraciones malignas frente al control (CA125: 1,15 pMol/L; HE4: 1,40 U/ml; ROMA: 5,92 \pm $1,71)$.

- Cuando se evaluaron las combinaciones, en relación a los diferentes clasificaciones de los grupos que contenían las tumoraciones se observa que en las mujeres que presentan tumoraciones 
Esthela N. Tinoco-Moreno; Alex F. Vivas-Lara; María G. Acuña-Chong; Mónica G. Núñez-Nájera

malignas la combinación de HE4 + I. ROMA presenta los mejores resultados en relación a los parámetros evaluados, pero con una baja LR + (ND). En cambio las mujeres que presentan tumoraciones benignas en las cuales HE4 + I. ROMA presenta una alta sensibilidad (100\%), mientras que en la especificidad las combinaciones CA125 + HE4 y CA125 + I. ROMA muestran mejor resultados que la combinación de HE4 + I. ROMA ( $100 \%$ vs. 91\%), igualmente en el resultado de VPP las combinaciones CA125 + HE4 y CA125 + I. ROMA muestran mejor resultados que la combinación de HE4 + I. ROMA que ésta con valores de casi la mitad de sus otras combinaciones $(100 \%$ vs. $51 \%)$. Finalmente en el grupo control HE4 + I. ROMA presenta los mejores resultados de la evaluación de los parámetros.

\section{Discusión}

- $\quad$ Los resultados sugieren, que el uso combinado de HE4 + I. ROMA ó HE4 sólo sería un buen indicador para determinar el riesgo de cáncer ovárico, debido a que ambos comparten similares fortalezas en cuanto a la clasificación de las pacientes más propensas.

- Los resultados muestran que HE4 presenta un valor medio altamente diferenciable, que permite distinguir entre mujeres con masas pélvicas malignas de las benignas (7.19 versus 5.71; control 5.79), al contrario de CA125 e I. ROMA (5.72 versus 4.64; control 4.57 y 5.92 versus 2.29; control 3.50, respectivamente).

- Estos resultados y los publicados en diferentes estudios concuerdan en relación a la superioridad de HE4 frente a CA125 e I. ROMA solos. Li et al (2011) y LinJ, Qin J, Sangvatanakul V (2013).

- Los resultados mostraron que HE4 combinado con I. ROMA presentan mayor sensibilidad que las combinaciones CA125 + HE4 y CA125 + I. ROMA (86\% versus 64\%), mientras que en la 
especificidad muestra una ligera mayor sensibilidad HE4 + I. ROMA que CA125 + I. ROMA (97\% versus 93\%) al contrario de CA125 + HE4 (82\%), de igual forma HE4 + I. ROMA presentan mayores niveles de VPP, VPN, LR+ y la menor LR- $(95 \%, 91 \%, 27.43$ y 0.15 , respectivamente) a diferencia de las otras dos combinaciones de marcadores con índice indistintamente del estatus menopáusico.

- Estos resultados confirman los obtenidos por Li et al (2011) y LinJ, Qin J, Sangvatanakul V (2013) y difieren de los de Braicuet al (2014) mostrando que la inclusión del biomarcador HE4 al algoritmo, mejoraría la diferenciación del riesgo de cáncer ovárico, demostrando, que la combinación del biomarcador HE4 + I. ROMA asegura significativamente mejores resultados para diferenciar el riesgo de cáncer ovárico, así como diferenciar entre masas benignas y malignas.

- Los resultados muestran que empleando la prueba U de Mann-Whitney la mayoría de las combinaciones presentaron diferencias estadísticamente significativas excepto el marcador CA125, solo cuando se evalúan mujeres pre menopáusicas versus post menopáusicas la, cual no presentó diferencia significativa $(p: 0.30302)$.

- Los resultados concuerdan con los mostrados por Cho et al (2015) y Fujiwara et al (2015) en la cual se observa que HE4 e I. ROMA serían candidatos adecuados para mejorar la diferenciación de masas, y difieren de los de Anton et al (2012) en donde no encuentra diferencia entre los marcadores empleados.

\section{Conclusiones}

En conclusión, HE4 e I. ROMA en combinación o solo el HE4, sirven como biomarcadores eficientes para la diferenciación de masas pélvicas en estadios tempranos y si se adiciona el estatus menopáusico, afianzan los resultados obtenidos. 
El estudio demostró que conocer los valores referenciales de los biomarcadores y/o redefinirlos en relación a la población estudiada, permitió mayor exactitud al aplicar los algoritmos diagnósticos, en donde se combinan resultados de diferentes biomarcadores y condiciones fisiológicas de los pacientes.

La investigación además transparenta la necesidad de estudios multímetros, recomendables para ampliar la población estudiada, validar los resultados obtenidos y aplicar la inclusión del valor de HE4 dentro del algoritmo diagnóstico de cáncer ovárico, en el país ya que la inclusión de mayor número de centros y pacientes sería posible diseñar una guía de uso acorde a la realidad del país considerando la diversidad étnica (razas) que poseemos y que respondería diferente frente a los factores ambientales, sociales y de la enfermedad.

\section{Bibliografía}

Abraham J. (2010). OVA1 test for preoperative assessment of ovarian cancer. Community Oncol. 7:24951.

Anastasi E., Granato T., Falzarano R., Storelli P., Ticino A., Frati L, Panici P., and Porpora M. (2013). The use of HE4, CA125 and CA72-4 biomarkers for differential diagnosis between ovarian endometrioma and epithelial ovarian cancer. Journal of Ovarian Research, 6:44.

Anton C., Carvalho F., Oliveira E., Maciel G., Baracat E., Carvalho J. (2012). A comparison of CA125, HE4, risk ovarian malignancy algorithm (ROMA), and risk malignancy index (RMI) for the classification of ovarian masses. Clinics; 67(5):437-441.

Bandiera E., Romani C., Specchia C, Zanotti L., Galli C., Ruggeri G., Tognon G., Bignotti E., Tassi R., Odicino F., Caimi L., Sartori E., Santin A., Pecorelli S., and AntonellaRavaggi. (2011). Serum human epididymis protein 4 (HE4) and Risk for Ovarian Malignancy Algorithm (ROMA) as new diagnostic and prognostic tools for epithelial ovarian cancer management. Cancer Epidemiol Biomarkers Prev. December;20(12):2496-2506. doi:10.1158/1055-9965.EPI-11-0635.

Będkowska G., Ławicki S., Gacuta E., Pawłowski P., and Szmitkowski M. (2015). M-CSF in a new biomarker panel with HE4 andCA 125 in the diagnostics of epithelial ovarian cancer patients. Journal of Ovarian Research, 8:27 
Braicu E., van Gorp T., Nassir M., Richter R., Chekerov R., Gasimli K., Timmerman D.,Vergote I. and Sehouli J. (2014). Preoperative HE4 and ROMA values do not improvethe CA125 diagnostic value for borderline tumors of the ovary (BOT) - a study of the TOC Consortium. Journal of Ovarian Research, 7:49.

Chen W.,Gao X., Han X., Zheng H., Guo L., Lu R. (2014). HE4 as a Serum Biomarker for ROMA Prediction and Prognosisof Epithelial Ovarian Cancer. Asian Pac J Cancer Prev, 15 (1), 101-105.

Chen Y., Chen Q., Liu Q., Gao F. (2016). Human epididymis protein 4 expression positively correlated with miR-21 and served as a prognostic indicator in ovarian cancer. Tumor Biology

Cho FN, Liu CB, Li JY, Chen SN, Yu KJ. (2012). Dramatic changes of CA 125 levels in apregnant woman with a degenerated subserosalmyoma. Taiwan J ObstetGynecol;51:117e8.

Cho H.,Park S., Park Y., Kim H., Kang J., Hong S., and Kyung M. (2015). Comparison of HE4, CA125, and Risk of Ovarian Malignancy Algorithm in the Prediction of Ovarian Cancer in Korean Women. J Korean Med Sci; 30: 1777-1783.

Drapkin R, von Horsten HH, Lin Y, Mok SC, Crum CP, Welch WR, et al. (2005). Humanepididymis protein 4 (HE4) is a secreted glycoprotein that is overexpressed byserous and endometrioid ovarian carcinomas. Cancer Res;65:2162e9.

Escudero JM, Auge JM, Filella X, Torne A, Pahisa J, Molina R. (2011). Comparison of serum human epididymis protein 4 with cancer antigen 125 as a tumor marker in patients with malignant and nonmalignant diseases. Clin Chem. 57:153444.

Fawzy A., Mohamed M., Ali M., Abd El-Magied M., Helal A. (2016). Tissue CA125 and HE4 Gene Expression Levels Offer Superior Accuracy in Discriminating Benign from Malignant Pelvic Masses. Asian Pac J Cancer Prev, 17 (1), 323-333

Ferlay J, Soerjomataram I, Ervik M, Dikshit R, Eser S, Mathers C et al. (2013). GLOBOCAN 2012 v1.0, Cancer Incidence and Mortality Worldwide: IARC Cancer Base No. 11[Internet]. Lyon, France: International Agency for Research on Cancer;.Available from: http://globocan.iarc.fr.

Fujiwara H., Suzuki M., Takeshima N., Takizawa K., Kimura E., Nakanishi T., Yamada K., Takano H., Sasaki H., Koyama K., Ochiai K. (2015). Evaluation of human epididymis protein 4 (HE4) and Riskof Ovarian Malignancy Algorithm (ROMA) as diagnostic tools of type I and type II epithelial ovarian cancer in Japanese women. Tumor Biol. 36:1045-1053

Gao L, Cheng HY, Dong L, Ye X, Liu YN, Chang XH, et al. (2011). The role of HE4 in ovarian cancer: inhibiting tumour cell proliferation and metastasis. J Int Med Res. 39:164560.

Gislefoss R., Langseth H., Bolstad N., Nustad K., and Mørkrid L. (2015). HE4 as an Early Detection Biomarker of EpithelialOvarian Cancer. Int J Gynecol Cancer 25: 1608-1615

Hellstrom I, Hellstrom KE. (2011). Two novel biomarkers, mesothelin and HE4, fordiagnosis of ovarian carcinoma. Expert Opin Med Diagn;5:227e40. 
Holschneider CH, Berek JS. (2000). Ovarian cancer: epidemiology, biology, and prognostic factors. SeminSurg Oncol.19:3-10.

Jacobs I, Oram D, Fairbanks J, Turner J, Frost C, Grudzinskas JG. (1990). A risk of malignancy index incorporating $\mathrm{CA} 125$, ultrasound and menopausal status for the accurate preoperative diagnosis of ovarian cancer. Br J ObstetGynaecol. 97:9229.

Jemal A, Siegel R, Ward E, Hao Y, Xu J, Thun MJ. (2009). Cancer statistics, 2009. CA Cancer J Clin. 59:22549.

Kondalsamy-Chennakesavan S, Hackethal A, Bowtell D.(2013). Australian OvarianCancer Study Group, Obermair A. Differentiating stage 1 epithelial ovariancancer from benign ovarian tumours using a combination of tumour markersHE4, CA125, and CEA and patient's age. Gynecol Oncol;129:467e71.

Leandersson P., Kalapotharakos G., Henic E., Borgfeldt H., Petzold M., Høyer-Hansen G. and Borgfeldt C. (2016). A biomarker panel increases the diagnostic performance for epithelial ovarian cancer type I and II in young women. Anticancer Research 36: 957-966.

Li F, Tie R, Chang K, Wang F, Deng S, Lu W, et al. (2012). Does risk for ovarian malignancy algorithm excel human epididymis protein 4 and CA125 in predicting epithelial ovarian cancer: a meta-analysis. BMC Cancer;12:258.

Lin J, Qin J, Sangvatanakul V. (2013). Human epididymis protein 4 for differentialdiagnosis between benign gynecologic disease and ovarian cancer: a systematic review and meta-analysis. Eur J ObstetGynecolReprod Biol;167:81e5.

Michalak M., Gasiorowska E., Markwitz E. (2015). Diagnostic value of CA125, HE4, ROMA and logistic regression model in pelvic mass diagnostics - our experience. Ginekol Pol. 86, 256-261

Moore RG, McMeekin DS, Brown AK, DiSilvestro P, Miller MC, Allard WJ, et al. (2009). A novel multiple marker bioassay utilizing HE4 and CA125 for the prediction of ovarian cancer in patients with a pelvic mass. GynecolOncol. 112:406.

Nunes N, Yazbek J, Ambler G, Hoo W, Naftalin J, Jurkovic D. (2012). Prospective evaluation of the IOTA logistic regression model LR2 for the diagnosis of ovarian cancer. Ultrasound Obstet Gynecol. 40:3559

Paek J, Lee SH, Yim GW, Lee M, Kim YJ, Nam EJ, et al. (2011). Prognostic significance ofhuman epididymis protein 4 in epithelial ovarian cancer. Eur J ObstetGynecolReprodBiol;158:338e42.

Pitynski K., Sporek A., Lipinska I., Banas T., Ludwin A., BałajewiczNowak M. (2015). Significance of adding progesterone to the Risk of Ovarian Malignancy Algorithm for early stage ovarian cancer detection in patients with a pelvic mass: A single-center caseecontrol study. Taiwanese Journal of Obstetrics \& Gynecology 54, 766e772. 
Romagnolo C., Leon A., Fabricio A., Taborelli M., Polesel J., Del Pup L., Steffan A., Cervo S., Ravaggi A., Zanotti L., Bandiera E., Odicino F., Scattolo N., Squarcina E., Papadakis C., Maggino T., Gion M. (2016). HE4, CA125 and risk ofovarian malignancy algorithm (ROMA) as diagnostic tools for ovarian cancer in patientswith a pelvic mass: An Italian multicenter study, Gynecologic Oncology, doi:10.1016/j.ygyno.2016.01.016

Rosen DG, Wang L, Atkinson JN, Yu Y, Lu KH, Diamandis EP, et al. (2005). Potentialmarkers that complement expression of CA125 in epithelial ovarian cancer.Gynecol Oncol;99:267e77.

Sánchez, S. E. (2014). Actualización en la epidemiología de la pre eclampsia: update. Revista Peruana de Ginecología y Obstetricia, 60(4), 309-320.

Siegel R, Naishadham D, Jemal A. (2012). Cancer statistics, 2012. CA Cancer J Clin.; 62:10-29. 